\title{
Combining linear and nonlinear diffusion
}

\author{
Manuel Delgado ${ }^{1}$ \\ Dpto. de Ecuaciones Diferenciales y Análisis Numérico \\ Universidad de Sevilla \\ Calle Tarfia s/n \\ 41012-Sevilla, Spain \\ e-mail: madelgado@us.es \\ Julián López-Gómez ${ }^{2}$ \\ Departamento de Matemática Aplicada \\ Universidad Complutense de Madrid \\ 28040-Madrid, Spain \\ e-mail: Lopez_Gomez@mat.ucm.es \\ Antonio Suárez ${ }^{1}$ \\ Dpto. de Ecuaciones Diferenciales y Análisis Numérico \\ Universidad de Sevilla \\ Calle Tarfia s/n \\ 41012-Sevilla, Spain \\ e-mail: suarez@us.es
}

\footnotetext{
${ }^{1}$ Supported by the Spanish Ministry of Science and Technology under Grants BFM2000-0797 and BFM2003-06446.

${ }^{2}$ Supported by the Spanish Ministry of Science and Technology under Grants BFM2000-0797 and REN2003-00707.
} 


\begin{abstract}
In this paper we study a generalized porous medium equation where the diffusion rate, say $\mathfrak{m}(x)$-spatially heterogeneous -, is assumed to be linear, $\mathfrak{m}=1$, on a piece of the support domain, $\Omega_{1}$, and slow nonlinear, $\mathfrak{m}(x)>1$, in its complement, $\Omega_{m}:=\Omega \backslash$ $\bar{\Omega}_{1}$. Most precisely, we characterize the existence of positive solutions and construct the corresponding global bifurcation diagram as one of the parameters of the model changes, showing that a continuous transition occurs between the diagrams of the completely linear case $\left(\Omega=\Omega_{1}\right)$ and of the completely nonlinear case $\left(\Omega_{m}=\Omega\right)$. As a result, the effect of a localized slow diffusion rate with varying support is completely characterized. Our analysis is imperative in order to design porous media multi-components systems with changing diffusion rates.
\end{abstract}

Key Words. Nonlinear diffusion. Spatial heterogeneities. From linear to nonlinear diffusion.

AMS Classification. 35B32, 35J25, 35J60, 35K57. 


\section{Introduction}

In this paper we study the positive solutions of the following boundary value problem

$$
\begin{cases}-\Delta\left(w^{\mathfrak{m}(x)}\right)=\lambda w & \text { in } \quad \Omega \\ w=0 & \text { on } \partial \Omega\end{cases}
$$

where $\Omega \subset \mathbb{R}^{N}, N \geq 1$, is a bounded domain of class $\mathcal{C}^{2}, \lambda \in \mathbb{R}$, and

$$
\mathfrak{m}=1+p \chi_{\Omega_{m}}
$$

where $\Omega_{m}$ is an open smooth subdomain of $\Omega$ such that $\bar{\Omega}_{m} \subset \Omega$ and $p \in \mathcal{C}\left(\bar{\Omega}_{m}\right)$ satisfies $p(x)>0$ for each $x \in \Omega_{m}$. Finally, we denote by

$$
\Omega_{1}:=\Omega \backslash \bar{\Omega}_{m}
$$

the open set where $\mathfrak{m}=1$. Given any measurable set $M \subset \Omega, \chi_{M}$ stands for the characteristic function of $M$, i.e., $\chi_{M}(x)=1$ for each $x \in M$, and $\chi_{M}(x)=0$ for each $x \in \Omega \backslash M$. An admissible choice for $p$ would be taking a constant $p>0$. Then,

$$
\mathfrak{m}=\chi_{\Omega_{1}}+(1+p) \chi_{\Omega_{m}} .
$$

In Figure 1, we have represented an admissible configuration. The dark region stands for $\Omega_{m}$, where $\mathfrak{m}>1$, and the white region is the subdomain of $\Omega$ where $\mathfrak{m}=1$.

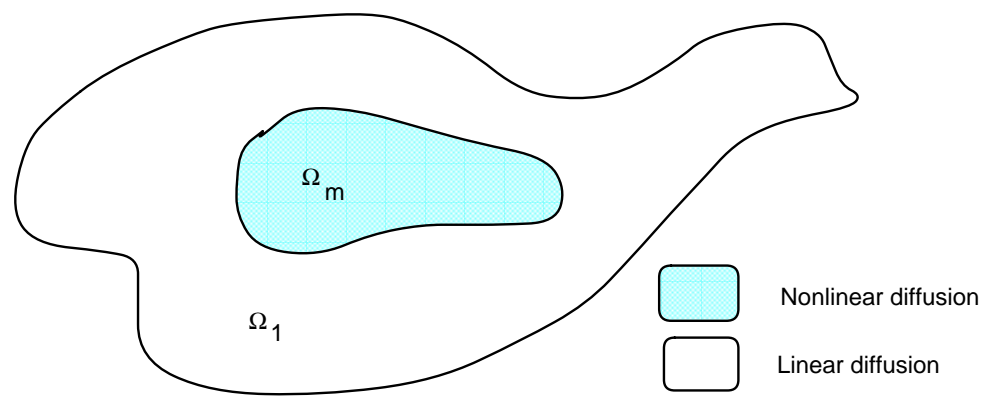

Figure 1. An admissible configuration.

In the special case $\Omega_{m}=\emptyset$, (1.1) reduces to the classical linear eigenvalue problem for the Laplacian under Dirichlet boundary conditions in $\Omega$. Subsequently, for any potential $V \in L^{\infty}(\Omega)$ we shall denote by $\sigma[-\Delta+V ; \Omega]$ the principal eigenvalue of $-\Delta+V$ in $\Omega$ under homogeneous Dirichlet boundary conditions. According to Krein-Rutman theorem, (1.1) possesses a positive solution if, and only, if $\lambda=\sigma[-\Delta ; \Omega]$. Actually, in such case, all positive solutions are multiples of a principal eigenfunction. 
On the other hand, when $\Omega_{m}=\Omega$ and $\mathfrak{m}$ is constant, (1.1) provides us with the classical porous medium equation, which generated a huge industry in Partial Differential Equations since the pioneering studies of G. I Barenblatt [2] and A. G. Aronson \& L. A. Peletier [1]. In fact, one of the results of [1] establishes that (1.1) possesses a positive solution if, and only if, $\lambda>0$, and that it is unique and asymptotically stable if it exists. Actually, if we denote it by $w_{\lambda}$ it turns out that $\lim _{\lambda \downarrow 0} w_{\lambda}=0$ and that $\lambda \mapsto w_{\lambda}$ is increasing (cf. [4] for further details). In Figure 2, we have represented a bifurcation diagram scheme of the positive solutions of (1.1) in these extreme opposite cases. Figure 2(a) shows the bifurcation diagram for the linear eigenvalue problem, and Figure 2(b) represents the bifurcation diagram of positive solutions for the classical porous medium equation. In Figure 2(a) we have denoted $\sigma_{1}:=\sigma[-\Delta ; \Omega]$.

Our main interest in this paper is focused into the problem of analyzing how change these diagrams when diffusion is nonlinear in some piece of $\Omega, \Omega_{m}$, whereas it is linear in the complement, $\Omega_{1}$, trying to ascertain all possible intermediate eventual transitions between the previous two limiting cases. Such an analysis is imperative in order to study the effect of local nonlinear diffusivities in the global dynamics of porous media. Consequently, we will throughout assume that $\Omega_{m}$, and so $\Omega_{1}$, are proper subdomains of $\Omega$. It should be noted that, though the non-linearity is discontinuous, it is of Caratheodory in $L^{\infty}$ and, hence, all solutions live in $W^{1, p}$ for all $p>1$.

The analysis of this problem fits into our general program of analyzing reaction diffusion equations in the presence of spatial heterogeneities; those heterogeneities might arise in nonlinear diffusion rates, of course. As it will become clear later the global nature of the corresponding bifurcation diagram of positive solutions of the general problem we are dealing with is rather different.

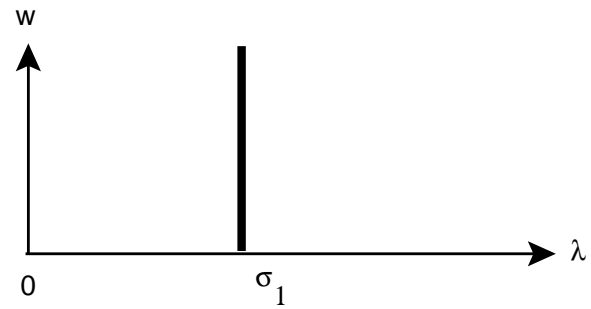

(a)

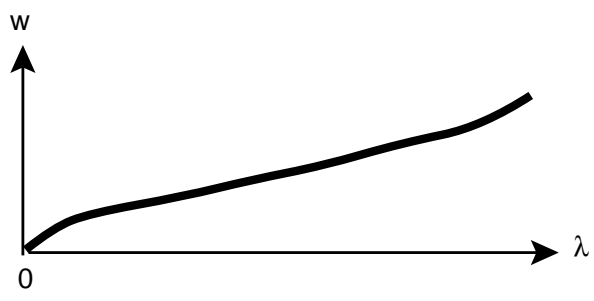

(b)

Figure 2. Bifurcation diagram in the limiting cases.

Since the change of variable

$$
u=w^{\mathfrak{m}(x)}
$$

transforms (1.1) into

$$
\begin{cases}-\Delta u=\lambda u^{\frac{1}{\mathfrak{m}(x)}} & \text { in } \Omega, \\ u=0 & \text { on } \partial \Omega .\end{cases}
$$


most of our attention will be focused into (1.2). By elliptic regularity theory, it is folklore that any weak non-negative solution $u \neq 0$ is an strong solution almost everywhere twice differentiable and, as a result of the maximum principle, $u(x)>0$ for each $x \in \Omega$ and $\frac{\partial u}{\partial n}(x)<0$ for each $x \in \partial \Omega$, where $n$ stands for the outward normal vector-field of $\Omega$. Therefore, a necessary condition for the existence of a positive weak solution is $\lambda>0$.

The following function will play a crucial role in our exposition

$$
\mu(\lambda):=\sigma\left[-\Delta-\lambda \chi_{\Omega_{1}} ; \Omega\right], \quad \lambda \in[0, \infty) .
$$

It satisfies $\mu(0)>0$, and, due to the monotonicity of the principal eigenvalue with respect to the potential, it is decreasing in $\lambda$. Actually, it satisfies $\mu^{\prime}(\lambda)<0$ for each $\lambda>0$, since $\lambda \mapsto \mu(\lambda)$ is concave; by a celebrated theorem of P. Hess and T. Kato [6] (cf. [8] for further details). Moreover, by the monotonicity of the principal eigenvalue with respect to the domain, given a ball $B \subset \Omega_{1}$ for each $\lambda \geq 0$ we have that

$$
\mu(\lambda)<\sigma\left[-\Delta-\lambda \chi_{\Omega_{1}} ; B\right]=\sigma[-\Delta ; B]-\lambda .
$$

Thus,

$$
\lim _{\lambda \uparrow \infty} \mu(\lambda)=-\infty
$$

and, hence, there exists

$$
\lambda_{0}=\lambda_{0}\left(\Omega_{1}\right) \in\left(0, \sigma\left[-\Delta ; \Omega_{1}\right]\right)
$$

such that

$$
\mu^{-1}(0) \cap[0, \infty)=\left\{\lambda_{0}\right\}
$$

Actually,

$$
\lambda_{0}\left(\Omega_{1}\right)>\sigma[-\Delta ; \Omega]
$$

since $\mu(\sigma[-\Delta ; \Omega])>0$. Indeed, if we denote by $\varphi$ a principal eigenfunction associated with $\sigma[-\Delta, \Omega]$, then

$$
\left(-\Delta-\sigma[-\Delta ; \Omega] \chi_{\Omega_{1}}\right) \varphi=\sigma[-\Delta ; \Omega]\left(1-\chi_{\Omega_{1}}\right) \varphi>0
$$

and, thanks to $[8$, Theorem 2.5], it is apparent that $\mu(\sigma[-\Delta ; \Omega])>0$. Moreover, as a result of the classical theory of P. Hess and T. Kato, $\mu(\lambda)$ is real analytic and concave (e.g., [6] and [8]). Once introduced these notations, we can state our main results. The next one provides us with the bifurcation diagram of positive solutions.

Theorem 1.1 Problem (1.2) possesses a positive solution if, and only if,

$$
0<\lambda<\lambda_{0}
$$

and it is unique if it exists. Moreover, if we denote it by $\theta_{\lambda}$, then, for each $\alpha \in(0,1)$, the map $\lambda \mapsto \theta_{\lambda}$ is increasing and of class $\mathcal{C}^{1}\left(\left(0, \lambda_{0}\right) ; \mathcal{C}_{0}^{\alpha}(\bar{\Omega})\right)$. Further,

$$
\lim _{\lambda \downarrow 0}\left\|\theta_{\lambda}\right\|_{\mathcal{C}^{1+\alpha}(\bar{\Omega})}=0 \quad \text { and } \quad \lim _{\lambda \uparrow \lambda_{0}}\left\|\theta_{\lambda}\right\|_{\mathcal{C}(K)}=\infty,
$$

for any compact subset $K \subset \Omega$. 
In Figure 3 we have represented the corresponding diagram of positive solutions of (1.2).

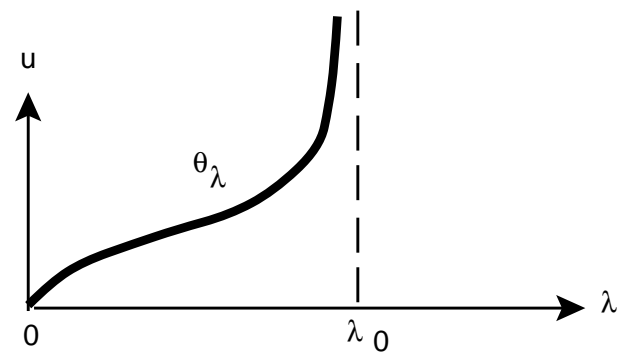

Figure 3. Bifurcation diagram in the general case.

The bifurcation diagram consists of an increasing differentiable curve emanating from $u=0$ at $\lambda=0$ and blowing-up to infinity, everywhere in $\Omega$, as $\lambda \uparrow \lambda_{0}$. It should be noted that the $u$-bifurcation diagrams for (1.2) look similar to those shown in Figure 2 for (1.1).

The next result establishes the existence of an homotopy between the two limiting bifurcation diagrams of Figure 2 and the bifurcation diagram of Figure 3. The concept of domain convergence used in its formulation is the one introduced in [8], for which there is continuous dependence of the principal eigenvalue and of the normalized principal eigenfunction in $W_{0}^{1,2}$.

Theorem 1.2 Let $m \in(1, \infty)$ and $\left\{\Omega_{m}^{\varepsilon}\right\}_{\{0<\varepsilon \leq 1\}}$ a monotone family of $\mathcal{C}^{2}$ subdomains of $\Omega$ such that

$$
\Omega_{m}^{1}=\Omega_{m} \quad \text { and } \quad \Omega_{1}^{\varepsilon}:=\Omega \backslash \bar{\Omega}_{m}^{\varepsilon}, \quad 0<\varepsilon \leq 1 .
$$

Set

$$
\mathfrak{m}_{\varepsilon}=\chi_{\Omega_{1}^{\varepsilon}}+m \chi_{\Omega_{m}^{\varepsilon}}, \quad 0<\varepsilon \leq 1
$$

and denote by $\theta_{[\lambda, \varepsilon]}, 0<\lambda<\lambda_{0}\left(\Omega_{1}^{\varepsilon}\right), 0<\varepsilon \leq 1$, the unique positive solution of

$$
\begin{cases}-\Delta u=\lambda u^{\frac{1}{\mathfrak{m}_{\varepsilon}(x)}} & \text { in } \Omega, \\ u=0 & \text { on } \partial \Omega .\end{cases}
$$

Then, the following assertions are true:

(a) If $\lim _{\varepsilon \downarrow 0} \Omega_{1}^{\varepsilon}=\Omega$, then

$$
\lim _{\varepsilon \downarrow 0} \lambda_{0}\left(\Omega_{1}^{\varepsilon}\right)=\sigma[-\Delta ; \Omega],
$$

and, for each $\lambda \in\left(\sigma[-\Delta ; \Omega], \lambda_{0}\left(\Omega_{1}\right)\right)$, there exists a unique $\varepsilon_{0} \in(0,1)$ such that $\lambda_{0}\left(\Omega_{1}^{\varepsilon_{0}}\right)=\lambda$. Moreover, $\lim _{\varepsilon \downarrow \varepsilon_{0}} \theta_{[\lambda, \varepsilon]}=\infty$ uniformly on compact subsets of $\Omega$ and

$$
\theta_{[\lambda, \varepsilon]}=\left\|\theta_{[\lambda, \varepsilon]}\right\|_{\mathcal{C}(\bar{\Omega})} \Phi_{\lambda}+o\left(\left\|\theta_{[\lambda, \varepsilon]}\right\|_{\mathcal{C}(\bar{\Omega})}\right) \quad \text { as } \varepsilon \downarrow \varepsilon_{0} \quad \text { in } \mathcal{C}^{1+\alpha}(\bar{\Omega})
$$

where $\Phi_{\lambda}$ stands for the principal eigenfunction of $\sigma\left[-\Delta-\lambda \chi_{\Omega_{1}^{\varepsilon_{0}}} ; \Omega\right]$ normalized so that $\left\|\Phi_{\lambda}\right\|_{\mathcal{C}(\bar{\Omega})}=1$, while

$$
\lim _{\varepsilon \downarrow 0}\left\|\theta_{[\lambda, \varepsilon]}\right\|_{\mathcal{C}(\bar{\Omega})}=0
$$

if $\lambda \in(0, \sigma[-\Delta, \Omega])$. 
(b) If $\lim _{\varepsilon \downarrow 0} \Omega_{m}^{\varepsilon}=\Omega$, then

$$
\lim _{\varepsilon \downarrow 0} \lambda_{0}\left(\Omega_{1}^{\varepsilon}\right)=\infty
$$

and, for each $\lambda \in(0, \infty)$,

$$
\lim _{\varepsilon \downarrow 0}\left\|\theta_{[\lambda, \varepsilon]}-\Theta_{\lambda}\right\|_{\mathcal{C}(\bar{\Omega})}=0
$$

where $\Theta_{\lambda}$ stands for the unique positive solution of the classical porous media equation ((1.2) with $\left.\Omega_{m}=\Omega\right)$.

The distribution of this paper is the following. In Section 2 we include the proof of Theorem 1.1 and analyze the asymptotic behavior of the positive solutions of the parabolic counterpart of (1.2). Finally, in Section 3 we prove Theorem 1.2.

\section{Proof of Theorem 1.1}

Subsequently, we denote by $P$ the cone of positive functions of $\mathcal{C}_{0}^{1+\alpha}(\bar{\Omega}) ; \stackrel{\circ}{P}$ standing for its interior. Given $u, v \in \mathcal{C}^{1+\alpha}(\bar{\Omega})$, it is said that $u>v$ if $u-v \in P \backslash\{0\}$, and $u \gg v$ if $u-v \in \stackrel{\circ}{P}$.

We already know that $\lambda>0$ is necessary for the existence of a positive solution. Now, let $\varphi_{\lambda} \gg 0$ denote a principal eigenfunction associated to $\mu(\lambda)(\mathrm{cf} .(1.3))$ and asume that (1.2) possesses a positive solution, $u$. Then, multiplying (1.2) by $\varphi_{\lambda}$, and integrating in $\Omega$ it is apparent that

$$
\mu(\lambda) \int_{\Omega} u \varphi_{\lambda}=\lambda \int_{\Omega_{m}} u^{\frac{1}{\mathfrak{m}}} \varphi_{\lambda}
$$

Thus, $\mu(\lambda)>0$ and, therefore, $\lambda<\lambda_{0}\left(\Omega_{1}\right)$. Recall that $\mu(\lambda)>0$ if and only if $0<\lambda<$ $\lambda_{0}\left(\Omega_{1}\right)$. This shows that $(1.5)$ is necessary for the existence.

To show that (1.5) implies the existence of a positive solution we use the sharp version of the method of sub and supersolutions developed by P. Hess [5] which demands no regularity assumptions. Suppose (1.5) and consider

$$
\tilde{\psi}:= \begin{cases}\psi & \text { in } \bar{B}, \\ 0 & \text { in } \Omega \backslash B,\end{cases}
$$

where $B$ is a ball with $\bar{B} \subset \Omega_{m}$ and $\psi$ stands for the positive eigenfunction associated to $\sigma[-\Delta ; B]$ normalized so that $\|\psi\|_{\mathcal{C}(\bar{B})}=1$. It is routine to check that the function

$$
\underline{u}:=\varepsilon \tilde{\psi}
$$

is a weak subsolution of (1.2) if

$$
0<\varepsilon \leq \min \left\{1,\left(\frac{\lambda}{\sigma[-\Delta ; B]}\right)^{\frac{\inf _{B} \mathfrak{m}}{\inf _{B} \mathfrak{m}-1}}\right\},
$$


since $\frac{\partial \psi}{\partial n}<0$ on $\partial B$, where $n$ is the outward unit normal vector-field of $B$. It should be noted that

$$
\inf _{B} \mathfrak{m}>1
$$

Actually, $\underline{u}$ provides us with a subsolution for any $\lambda>0$.

Now, pick $\lambda \in\left(0, \lambda_{0}\right)$ and, for each sufficiently small $\delta>0$, consider

$$
\Omega_{m, \delta}:=\left\{x \in \Omega_{m}: \operatorname{dist}\left(x, \partial \Omega_{m}\right)>\delta\right\},
$$

and

$$
\mu^{\delta}(\lambda):=\sigma\left[-\Delta-\lambda \chi_{\Omega_{1, \delta}} ; \Omega\right]
$$

where

$$
\Omega_{1, \delta}:=\Omega \backslash \bar{\Omega}_{m, \delta} .
$$

By the continuous dependence of the principal eigenvalue with respect to the potential, $\mu^{\delta}(\lambda)>0$ if $\delta>0$ is sufficiently small. Assume $\delta$ has been chosen in that way. Let $\varphi_{\lambda}^{\delta}$ denote the positive eigenfunction associated to $\mu^{\delta}(\lambda)$ normalized so that $\left\|\varphi_{\lambda}^{\delta}\right\|_{\mathcal{C}(\bar{\Omega})}=1$. Then, the function

$$
\bar{u}:=K \varphi_{\lambda}^{\delta}
$$

provides us with a positive supersolution of (1.2) if

$$
K \geq \max \left\{1,\left[\frac{\lambda}{\mu^{\delta}(\lambda)}\left(\inf _{\Omega_{m, \delta}} \varphi_{\lambda}^{\delta}\right)^{\frac{1-\sup _{\Omega_{m}, \delta} \mathfrak{m}}{\sup _{\Omega_{m}, \delta}}}\right]^{\frac{\inf _{\Omega_{m, \delta}} \mathfrak{m}}{\inf _{\Omega_{m, \delta}} m-1}},\left(\inf _{\Omega_{m}} \varphi_{\lambda}^{\delta}\right)^{-1}\right\}
$$

Note that $\inf _{\Omega_{m, \delta}} \mathfrak{m}>1$. Finally, by choosing $\varepsilon>0$ sufficiently small and $K>1$ sufficiently large, it is clear that $\underline{u} \leq \bar{u}$ and, therefore, (1.2) possesses a weak positive solution in the interval $[\underline{u}, \bar{u}]$; necessarily strong, by elliptic regularity. This concludes the proof of the existence.

To prove the uniqueness we will adapt the argument given in the proof of $[4$, Theorem 3.2]. Suppose $u$ is a positive solution of (1.2). Then,

$$
\begin{cases}\left(-\Delta-\lambda u^{\frac{1}{\mathfrak{m}}-1}\right) u=0 & \text { in } \Omega, \\ u=0 & \text { on } \partial \Omega,\end{cases}
$$

and, hence, by the uniqueness of the principal eigenvalue, we find that

$$
\sigma\left[-\Delta-\lambda u^{\frac{1}{\mathfrak{m}}-1} ; \Omega\right]=0 .
$$

Suppose (1.2) possesses a further positive solution $v \neq u$. Then,

$$
-\Delta(u-v)=\lambda\left(u^{\frac{1}{\mathfrak{m}}}-v^{\frac{1}{\mathfrak{m}}}\right)=\frac{\lambda}{\mathfrak{m}} \int_{0}^{1}[t u+(1-t) v]^{\frac{1}{\mathfrak{m}}-1} d t(u-v) .
$$


Thus, setting

$$
W:=-\frac{\lambda}{\mathfrak{m}} \int_{0}^{1}[t u+(1-t) v]^{\frac{1}{\mathfrak{m}}-1} d t
$$

gives

$$
\begin{cases}(-\Delta+W)(u-v)=0 & \text { in } \Omega \\ u-v=0 & \text { on } \partial \Omega\end{cases}
$$

In $\Omega_{1}, W=-\lambda$, while, in $\Omega_{m}$,

$$
\int_{0}^{1}[t u+(1-t) v]^{\frac{1}{\mathfrak{m}}-1} d t<u^{\frac{1}{\mathfrak{m}}-1} \int_{0}^{1} t^{\frac{1}{\mathfrak{m}}-1} d t=\mathfrak{m} u^{\frac{1}{\mathfrak{m}}-1}
$$

and, hence,

$$
W>-\lambda u^{\frac{1}{\mathfrak{m}}-1} .
$$

Thus, by the monotonicity of the principal eigenvalue with respect to the potential, we find from (2.4) that

$$
\sigma[-\Delta+W ; \Omega]>0
$$

As the principal eigenvalue is dominant, from (2.5) it is apparent that $u=v$. This contradiction ends the proof of the uniqueness.

Subsequently, for each $\lambda \in\left(0, \lambda_{0}\right)$, we denote by $\theta_{\lambda}$ the unique positive solution of (1.2). The fact that the map

$$
\begin{array}{cc}
\left(0, \lambda_{0}\right) & \longrightarrow \mathcal{C}_{0}^{\alpha}(\bar{\Omega}) \\
\lambda & \mapsto \quad \theta_{\lambda}
\end{array}
$$

is of class $\mathcal{C}^{1}$ follows easily from the implicit function theorem applied to the operator

$$
\begin{array}{ccc}
\left(0, \lambda_{0}\right) \times \stackrel{\circ}{P} & \stackrel{T}{\longrightarrow} & \mathcal{C}_{0}^{\alpha}(\bar{\Omega}) \\
(\lambda, u) & \mapsto & u-\lambda(-\Delta)^{-1}\left(u^{\frac{1}{\mathfrak{m}}}\right)
\end{array}
$$

whose zeros are in one-to-one correspondence with the positive solutions of (1.2). $T$ is of class $\mathcal{C}^{1}$ and, for each $\lambda \in\left(0, \lambda_{0}\right)$,

$$
D_{u} T\left(\lambda, \theta_{\lambda}\right): \mathcal{C}_{0}^{1+\alpha}(\bar{\Omega}) \longrightarrow \mathcal{C}_{0}^{\alpha}(\bar{\Omega})
$$

is the linear continuous compact operator defined by

$$
D_{u} T\left(\lambda, \theta_{\lambda}\right) u:=u-\lambda(-\Delta)^{-1}\left(\frac{1}{\mathfrak{m}} \theta_{\lambda}^{\frac{1}{\mathfrak{m}}-1} u\right), \quad u \in \mathcal{C}_{0}^{1+\alpha}(\bar{\Omega})
$$


Notice that $\theta_{\lambda}(x)>0$ for each $x \in \Omega_{m}$. Recall that $\Omega_{m}$ is the region where $\mathfrak{m}(x)>1$, and hence $\frac{1}{\mathfrak{m}(x)}-1<0$. Since $D_{u} T\left(\lambda, \theta_{\lambda}\right)$ is Fredholm of index zero, it is an isomorphism if $u=0$ is the unique function $u \in \mathcal{C}_{0}^{1+\alpha}(\bar{\Omega})$ such that

$$
u=\lambda(-\Delta)^{-1}\left(\frac{1}{\mathfrak{m}} \theta_{\lambda}^{\frac{1}{\mathfrak{m}}-1} u\right)
$$

By elliptic regularity any of these functions provides us with a strong solution of

$$
\begin{cases}\left(-\Delta-\frac{\lambda}{\mathfrak{m}} \theta_{\lambda}^{\frac{1}{\mathfrak{m}}-1}\right) u=0 & \text { in } \Omega, \\ u=0 & \text { on } \partial \Omega .\end{cases}
$$

Thanks to (2.4), the monotonicity of the principal eigenvalue with respect to the potential gives

$$
\sigma\left[-\Delta-\frac{\lambda}{\mathfrak{m}} \theta_{\lambda}^{\frac{1}{\mathfrak{m}}-1} ; \Omega\right]>\sigma\left[-\Delta-\lambda \theta_{\lambda}^{\frac{1}{\mathfrak{m}}-1} ; \Omega\right]=0,
$$

since $\frac{1}{\mathfrak{m}}<1$. Hence, by the dominance of the principal eigenvalue, $u=0$ is the unique solution of $(2.7)$, and, consequently, $D_{u} T\left(\lambda, \theta_{\lambda}\right)$ is a linear isomorphism. Therefore, by the implicit function theorem, the map (2.6) is of class $\mathcal{C}^{1}$.

Now, by implicit differentiation with respect to $\lambda$ it is apparent that, for any $\lambda \in$ $\left(0, \lambda_{0}\right)$

$$
\begin{cases}\left(-\Delta-\frac{\lambda}{\mathfrak{m}} \theta_{\lambda}^{\frac{1}{\mathfrak{m}}-1}\right) \frac{d \theta_{\lambda}}{d \lambda}=\theta_{\lambda}^{\frac{1}{\mathfrak{m}}} & \text { in } \Omega, \\ \frac{d \theta_{\lambda}}{d \lambda}=0 & \text { on } \partial \Omega .\end{cases}
$$

Thanks to (2.8), the inverse of the differential operator in the left hand side of (2.9) is strongly positive. Thus, $\frac{d \theta_{\lambda}}{d \lambda} \in \stackrel{\circ}{P}$ and, hence, the map (2.6) is strongly increasing. In particular, $\lambda \mapsto \theta_{\lambda}$ is point-wise increasing in $\Omega$.

We now prove the validity of the second limit in (1.6). Since

$$
\frac{d \theta_{\lambda}}{d \lambda} \gg 0 \quad \text { and } \quad \chi_{\Omega_{1}}<\frac{1}{\mathfrak{m}} \theta_{\lambda}^{\frac{1}{\mathfrak{m}}-1}
$$

from (2.9) we find that

$$
\left(-\Delta-\lambda \chi_{\Omega_{1}}\right) \frac{d \theta_{\lambda}}{d \lambda}>\theta_{\lambda}^{\frac{1}{\mathfrak{m}}} \quad \text { in } \Omega
$$

Pick $\lambda_{*} \in\left(0, \lambda_{0}\right)$. Then, for each $\lambda \in\left(\lambda_{*}, \lambda_{0}\right)$, we have that

$$
\left(-\Delta-\lambda \chi_{\Omega_{1}}\right) \frac{d \theta_{\lambda}}{d \lambda}>\theta_{\lambda_{*}}^{\frac{1}{\mathfrak{m}}} \quad \text { in } \Omega,
$$

since $\theta_{\lambda}>\theta_{\lambda_{*}}$. 
On the other hand, since $\mu(\lambda)$ is a simple eigenvalue, a classical perturbation result coming from T. Kato [7] shows that the principal eigenfunctions $\varphi_{\lambda}$ associated to $\mu(\lambda)$ can be chosen so that the map

$$
\begin{aligned}
\mathbb{R} & \longrightarrow \mathcal{C}_{0}(\bar{\Omega}) \\
\lambda & \mapsto \varphi_{\lambda}
\end{aligned}
$$

be analytic. For such choice, there exist $C>0$ and a sufficiently small $\varepsilon>0$ such that, for each $\lambda \in\left[\lambda_{0}-\varepsilon, \lambda_{0}\right)$,

$$
\theta_{\lambda_{*}}^{\frac{1}{\mathfrak{m}}} \geq C \varphi_{\lambda_{0}}>\frac{C}{2} \varphi_{\lambda} \quad \text { in } \Omega
$$

Thus, substituting in $(2.10)$ gives

$$
\left(-\Delta-\lambda \chi_{\Omega_{1}}\right) \frac{d \theta_{\lambda}}{d \lambda}>\frac{C}{2} \varphi_{\lambda} \quad \text { in } \Omega, \quad \lambda \in\left[\lambda_{0}-\varepsilon, \lambda_{0}\right) .
$$

Since $\mu(\lambda)>0$, the operator on the left hand side of (2.11) has a strongly positive inverse, and, hence,

$$
\frac{d \theta_{\lambda}}{d \lambda}>\Theta_{\lambda}, \quad \lambda \in\left[\lambda_{0}-\varepsilon, \lambda_{0}\right)
$$

where $\Theta_{\lambda}$ is the unique strong solution of

$$
\left(-\Delta-\lambda \chi_{\Omega_{1}}\right) \Theta_{\lambda}=\frac{C}{2} \varphi_{\lambda} \quad \text { in } \Omega, \quad \lambda \in\left[\lambda_{0}-\varepsilon, \lambda_{0}\right),
$$

such that $\Theta_{\lambda}=0$ on $\partial \Omega$. Solving (2.13) yields to

$$
\Theta_{\lambda}=\frac{C}{2 \mu(\lambda)} \varphi_{\lambda}, \quad \lambda \in\left[\lambda_{0}-\varepsilon, \lambda_{0}\right)
$$

Since $\varphi_{\lambda}$ converges uniformly to $\varphi_{\lambda_{0}}$ as $\lambda \uparrow \lambda_{0}$,

$$
\lim _{\lambda \uparrow \lambda_{0}} \Theta_{\lambda}=\infty
$$

uniformly on compact subsets of $\Omega$. Further, since $\mu^{\prime}\left(\lambda_{0}\right)<0$ implies

$$
\lim _{\lambda \uparrow \lambda_{0}} \int_{\lambda_{0}-\varepsilon}^{\lambda} \frac{1}{\mu(s)} d s=\infty,
$$

integrating (2.12), the second limit of (1.6) follows.

The validity of the first limit of (1.6) follows from the fact that $\lim _{\lambda \downarrow 0} \theta_{\lambda}$ provides us with a non-negative solution of (1.2) at $\lambda=0$; necessarily zero. This concludes the proof of Theorem 1.1.

It should be noted that the uniqueness of the positive solution $\theta_{\lambda}$ can be obtained as well by a rather standard continuation argument based on the the fact that any positive 
solution of (1.2) is non-degenerate. It also follows from [3, Remark 1]; [3, Theorem 1] cannot be invoked since $u^{\frac{1}{\mathfrak{m}}-1}$ is not decreasing.

The proof of Theorem 1.1 also provides us with the asymptotic behaviour of the positive solutions of following parabolic problem

$$
\begin{cases}\partial_{t} u-\Delta u=\lambda u^{\frac{1}{\mathfrak{m}(x)}} & \text { in } \Omega \times(0, \infty), \\ u=0 & \text { on } \partial \Omega \times(0, \infty), \\ u(\cdot, 0)=u_{0}>0 & \text { in } \Omega,\end{cases}
$$

where $u_{0} \in \mathcal{C}(\bar{\Omega})$, though this regularity requirement might be considerably weakened. Since $\mathfrak{m}>1$, we have that $u^{\frac{1}{\mathfrak{m}}}<u$ in $\Omega$ in the region where $u>1$ and hence, for each $u_{0},(2.14)$ possesses a unique solution positively globally defined in time. Let $u_{\lambda}\left(x, t ; u_{0}\right)$ denote it. The following result provides us with the limiting behavior of $u_{\lambda}$ as $t \uparrow \infty$ accordingly to the value of the parameter $\lambda$.

Theorem 2.1 The following assertions are true:

(a) $u=0$ is a global attractor of (2.14) if $\lambda \leq 0$.

(b) $u=\theta_{\lambda}$ is a global attractor of (2.14) if $\lambda \in\left(0, \lambda_{0}\right)$.

(c) For any compact subset $K \subset \Omega$,

$$
\lim _{t \uparrow \infty} u_{\lambda}\left(\cdot, t ; u_{0}\right)=\infty \quad \text { uniformly in } K,
$$

$$
\text { if } \lambda \geq \lambda_{0} \text {. }
$$

Proof: Part (b) follows straight ahead from the theory of D. Sattinger [9] using that (1.2) possesses arbitrarily small subsolutions and arbitrarily large supersolutions and that $\theta_{\lambda}$ is the unique positive solution of (1.2). Part (a) follows comparing the solution of (2.14) for $\lambda \leq 0$ with its solutions for arbitrarily small $\lambda>0$. The result follows easily from the fact that $\lim _{\lambda \downarrow 0} \theta_{\lambda}=0$. The same argument easily adapts to show Part (c). Now, one should compare the solutions of (2.14) with the solutions of (2.14) for $\lambda<\lambda_{0}$ approaching to $\lambda_{0}$. Then, the result follows readily from the fact that $\lim _{\lambda \uparrow \lambda_{0}} \theta_{\lambda}=\infty$ uniformly on compact subsets of $\Omega$. This concludes the proof.

\section{Proof of Theorem 1.2}

\subsection{Proof of Part (a)}

Suppose $\left\{\Omega_{1}^{\varepsilon}\right\}_{\varepsilon \in(0,1]}$ is increasing and

$$
\lim _{\varepsilon \downarrow 0} \Omega_{1}^{\varepsilon}=\Omega
$$


Set

$$
\mu_{\varepsilon}(\lambda):=\sigma\left[-\Delta-\lambda \chi_{\Omega_{1}^{\varepsilon}} ; \Omega\right], \quad(\lambda, \varepsilon) \in[0, \infty) \times(0,1] .
$$

By the continuous dependence of the principal eigenvalue with respect to the potential, (3.1) implies

$$
\mu_{0}(\lambda):=\lim _{\varepsilon \downarrow 0} \mu_{\varepsilon}(\lambda)=\sigma[-\Delta ; \Omega]-\lambda, \quad \lambda \in[0, \infty)
$$

uniformly in compact intervals of the parameter $\lambda \in[0, \infty)$. Thus, the unique zero of $\mu_{\varepsilon}(\lambda)$ must approach the unique zero of $\mu_{0}(\lambda)$ as $\varepsilon \downarrow 0$, and hence (1.9) holds. Moreover, as $\varepsilon$ ranges from 0 to $1, \lambda_{0}\left(\Omega_{1}^{\varepsilon}\right)$ increases from $\sigma[-\Delta ; \Omega]$ up to reach the value $\lambda_{0}\left(\Omega_{1}\right)$. Thus, for any $\lambda \in\left(\sigma[-\Delta ; \Omega], \lambda_{0}\left(\Omega_{1}\right)\right)$ there exists a unique $\varepsilon_{0}=\varepsilon(\lambda) \in(0,1)$ such that

$$
\lambda=\lambda_{0}\left(\Omega_{1}^{\varepsilon(\lambda)}\right) .
$$

because of the monotonicity of $\mu_{\varepsilon}$, and hence of $\lambda_{0}\left(\Omega_{1}^{\varepsilon}\right)$. To prove (1.10) we argue as follows. Let $\varphi_{[\lambda, \varepsilon]}$ denote the positive eigenfunction associated to $\mu_{\varepsilon}(\lambda)$ normalized so that

$$
\left\|\varphi_{[\lambda, \varepsilon]}\right\|_{W_{0}^{1,2}(\Omega)}=1
$$

Then, multiplying the $\theta_{[\lambda, \varepsilon]}$-equation by $\varphi_{[\lambda, \varepsilon]}$, integrating in $\Omega$, applying the formula of integration by parts and using the definition of $\varphi_{[\lambda, \varepsilon]}$ it is easy to see that

$$
\mu_{\varepsilon}(\lambda) \int_{\Omega} \theta_{[\lambda, \varepsilon]} \varphi_{[\lambda, \varepsilon]}=\lambda \int_{\Omega_{m}^{\varepsilon}} \theta_{[\lambda, \varepsilon]}^{\frac{1}{m}} \varphi_{[\lambda, \varepsilon]} .
$$

Let $\varphi$ denote the principal eigenfunction of $\sigma[-\Delta ; \Omega]$ normalized so that $\|\varphi\|_{\mathcal{C}(\bar{\Omega})}=1$. Since $\lambda>\sigma[-\Delta ; \Omega]$, by a direct calculation it follows that there exists $\beta_{0}>0$ such that for each $\beta \in\left(0, \beta_{0}\right]$ and $\varepsilon \in\left[\varepsilon_{0}, 1\right]$ the function

$$
\underline{u}:=\beta \varphi
$$

is a subsolution of (1.8). As these equations admit arbitrarily large supersolutions above $\beta \varphi$, by the uniqueness of the positive solution we have that

$$
\theta_{[\lambda, \varepsilon]} \geq \beta_{0} \varphi \quad \text { in } \Omega \quad \forall \varepsilon \in\left[\varepsilon_{0}, 1\right] .
$$

Since $\Omega_{m}^{\varepsilon} \subset \Omega_{m}$ for each $\varepsilon \in(0,1], \bar{\Omega}_{m} \subset \Omega$, and $\varphi$ is bounded away from zero in $\Omega_{m}$, due to (3.4) there exists a constant $\omega>0$ such that

$$
\theta_{[\lambda, \varepsilon]} \geq \omega \quad \text { in } \Omega_{m}^{\varepsilon} \quad \forall \varepsilon \in\left[\varepsilon_{0}, 1\right]
$$

On the other hand, adapting the argument of the proof of [8, Theorem 4.2], it is easy to see that

$$
\lim _{\varepsilon \downarrow \varepsilon_{0}}\left\|\varphi_{[\lambda, \varepsilon]}-\varphi_{\left[\lambda, \varepsilon_{0}\right]}\right\|_{W_{0}^{1,2}(\Omega)}=0
$$


though it should be pointed out that here varies the potential, instead of the domain. Thus, (3.3) implies

$$
\lim _{\varepsilon \downarrow \varepsilon_{0}} \int_{\Omega} \theta_{[\lambda, \varepsilon]} \varphi_{[\lambda, \varepsilon]}=\infty
$$

since

$$
\lim _{\varepsilon \downarrow \varepsilon_{0}} \mu_{\varepsilon}(\lambda)=\mu_{\varepsilon_{0}}(\lambda)=\mu_{\varepsilon_{0}}\left(\lambda_{0}\left(\Omega_{1}^{\varepsilon_{0}}\right)\right)=0
$$

Consequently,

$$
\lim _{\varepsilon \downarrow \varepsilon_{0}}\left\|\theta_{[\lambda, \varepsilon]}\right\|_{\mathcal{C}(\bar{\Omega})}=\infty
$$

Let $\left\{\varepsilon_{n}\right\}_{n \geq 1}$ be any decreasing sequence such that $\lim _{n \rightarrow \infty} \varepsilon_{n}=\varepsilon_{0}$ and set

$$
\Theta_{n}:=\frac{\theta_{\left[\lambda, \varepsilon_{n}\right]}}{\left\|\theta_{\left[\lambda, \varepsilon_{n}\right]}\right\|_{\mathcal{C}(\bar{\Omega})}}, \quad n \geq 1 .
$$

These functions satisfy

$$
\left\|\Theta_{n}\right\|_{\mathcal{C}(\bar{\Omega})}=1, \quad \Theta_{n} \gg 0, \quad n \geq 1
$$

Moreover,

$$
\Theta_{n}=\lambda(-\Delta)^{-1}\left(\chi_{\Omega_{1}^{\varepsilon_{n}}} \Theta_{n}+\chi_{\Omega_{m}^{\varepsilon_{n}}} \Theta_{n}^{\frac{1}{m}}\left\|\theta_{\left[\lambda, \varepsilon_{n}\right]}\right\|_{\mathcal{C}(\bar{\Omega})}^{\frac{1-m}{m}}\right), \quad n \geq 1 .
$$

Thanks to (3.6), there exists a constant $C>0$ such that

$$
\left\|\chi_{\Omega_{1}^{\varepsilon_{n}}} \Theta_{n}+\chi_{\Omega_{m}^{\varepsilon_{n}}} \Theta_{n}^{\frac{1}{m}}\right\| \theta_{\left[\lambda, \varepsilon_{n}\right]}\left\|_{\mathcal{C}(\bar{\Omega})}^{\frac{1-m}{m}}\right\|_{\mathcal{C}(\bar{\Omega})} \leq C, \quad n \geq 1
$$

Thus, by the compactness of $(-\Delta)^{-1}$, there exists $\Theta \in \mathcal{C}_{0}(\bar{\Omega})$ such that, along some subsequence that we label again by $n$,

$$
\lim _{n \rightarrow \infty}\left\|\Theta_{n}-\Theta\right\|_{\mathcal{C}(\bar{\Omega})}=0
$$

Necessarily,

$$
\|\Theta\|_{\mathcal{C}(\bar{\Omega})}=1, \quad \Theta \geq 0
$$

and passing to the limit as $n \rightarrow \infty$ in (3.8) gives

$$
\Theta=\lambda(-\Delta)^{-1}\left(\chi_{\Omega_{1}^{\varepsilon_{0}}} \Theta\right)
$$

Thanks to (3.10), $\Theta=0$ in $\Omega$ if $\Theta=0$ in $\Omega_{1}^{\varepsilon_{0}}$, which is impossible by (3.9). Thus, $\Theta>0$ in $\Omega_{1}^{\varepsilon_{0}}$ and, hence, by the strong positivity of $(-\Delta)^{-1}, \Theta \gg 0$ in $\Omega$. Actually,

$$
\Theta=\frac{\varphi_{\left[\lambda, \varepsilon_{0}\right]}}{\left\|\varphi_{\left[\lambda, \varepsilon_{0}\right]}\right\|_{\mathcal{C}(\bar{\Omega})}} .
$$


As the previous process is independent on the sequence of $\varepsilon$ 's converging to $\varepsilon_{0}$, it follows that

$$
\lim _{\varepsilon \downarrow \varepsilon_{0}}\left\|\frac{\theta_{[\lambda, \varepsilon]}}{\left\|\theta_{[\lambda, \varepsilon]}\right\|_{\mathcal{C}(\bar{\Omega})}}-\frac{\varphi_{\left[\lambda, \varepsilon_{0}\right]}}{\left\|\varphi_{\left[\lambda, \varepsilon_{0}\right]}\right\|_{\mathcal{C}(\bar{\Omega})}}\right\|_{\mathcal{C}^{1+\alpha}(\bar{\Omega})}=0
$$

for any $\alpha \in(0,1)$. This completes the proof of $(1.10)$. Note that

$$
\Phi_{\lambda}:=\frac{\varphi_{\left[\lambda, \varepsilon_{0}\right]}}{\left\|\varphi_{\left[\lambda, \varepsilon_{0}\right]}\right\|_{\mathcal{C}(\bar{\Omega})}}
$$

Next, we prove (1.11). Suppose $0<\lambda<\sigma[-\Delta ; \Omega]$. Then,

$$
\lim _{\varepsilon \downarrow 0} \mu_{\varepsilon}(\lambda)=\sigma[-\Delta ; \Omega]-\lambda>0
$$

Moreover, thanks to (2.3) and due to the fact that

$$
\lim _{\varepsilon \downarrow 0}\left\|\frac{\varphi_{[\lambda, \varepsilon]}}{\left\|\varphi_{[\lambda, \varepsilon]}\right\|_{\mathcal{C}(\bar{\Omega})}}-\frac{\varphi_{[\lambda, 0]}}{\left\|\varphi_{[\lambda, 0]}\right\|_{\mathcal{C}(\bar{\Omega})}}\right\|_{\mathcal{C}(\bar{\Omega})}=0,
$$

there exists a constant $C>0$ such that $\theta_{[\lambda, \varepsilon]} \leq C$ for any sufficiently small $\varepsilon>0$, since $\Omega_{m}^{\varepsilon}$ is decreasing. Notice that $\varphi_{[\lambda, 0]}$ is a principal eigenfunction associated with $\sigma[-\Delta ; \Omega]$. Thus, passing to the limit as $\varepsilon \downarrow 0$ in (3.3) we find that

$$
\lim _{\varepsilon \downarrow 0} \int_{\Omega} \theta_{[\lambda, \varepsilon]} \frac{\varphi_{[\lambda, \varepsilon]}}{\left\|\varphi_{[\lambda, \varepsilon]}\right\|_{\mathcal{C}(\bar{\Omega})}}=0
$$

because $\lim _{\varepsilon \downarrow 0}\left|\Omega_{m}^{\varepsilon}\right|=0$. Since $\theta_{[\lambda, \varepsilon]}$ is uniformly bounded above by a constant for any sufficiently small $\varepsilon>0$, it is easy to see that (1.11) follows from (3.12) and (3.13).

\subsection{Proof of Part (b)}

Suppose $\lim _{\varepsilon \downarrow 0} \Omega_{m}^{\varepsilon}=\Omega$. Then,

$$
\lim _{\varepsilon \downarrow 0} \mu_{\varepsilon}(\lambda)=\sigma[-\Delta ; \Omega]
$$

uniformly in compact intervals of $\lambda \in[0, \infty)$. Thus, (1.12) holds true.

Fix $\lambda>0$ and let $\varepsilon_{0}=\varepsilon(\lambda)>0$ such that $\lambda_{0}\left(\Omega_{1}^{\varepsilon}\right)>\lambda$ for each $\varepsilon \in\left[0, \varepsilon_{0}\right)$. Thanks to Theorem 1.1 , for each $\varepsilon \in\left[0, \varepsilon_{0}\right)$, (1.8) possesses a unique positive solution, subsequently denoted by $\theta_{[\lambda, \varepsilon]}$. In order to get uniform a priori bounds for these positive solutions the estimate given by (2.3) cannot be used, since $\lim _{\varepsilon \downarrow 0} \inf _{\Omega_{m}^{\varepsilon}} \varphi_{[\lambda, \varepsilon]}=0$. Instead of using that estimate we slightly enlarge the domain $\Omega$. For each sufficiently small $\delta>0$ we consider

$$
\Omega^{\delta}:=\left\{x \in \mathbb{R}^{N}: \operatorname{dist}(x, \Omega)<\delta\right\} .
$$


Fix $\varepsilon<\varepsilon_{0}$. Thanks to the continuous dependence of the principal eigenvalue with respect to the domain, there exists $\delta_{0}=\delta(\lambda, \varepsilon)>0$ such that

$$
\mu_{\varepsilon}^{\delta}(\lambda):=\sigma\left[-\Delta-\lambda \chi_{\Omega_{1}^{\varepsilon}} ; \Omega^{\delta}\right]>0 \quad \text { if } \delta \in\left[0, \delta_{0}\right) .
$$

Fix one of those $\delta$ 's and let $\varphi_{\delta}$ denote the principal eigenfunction of $\mu_{\varepsilon}^{\delta}(\lambda)$ normalized so that $\left\|\varphi_{\delta}\right\|_{\mathcal{C}(\bar{\Omega})}=1$. A direct calculation shows that the function

$$
\bar{u}:=K \varphi_{\delta}
$$

provides us with a positive supersolution of (1.8) in $\Omega$ for each sufficiently small $\varepsilon>0$ and sufficiently large $K>1$, which can be chosen to be independent of $\varepsilon$. Notice that those supersolutions are bounded away from zero all over $\Omega$. Also, thanks to (2.2), all the corresponding positive solutions are bounded bellow by a universal positive function -bellow the supersolution. By adapting the compactness argument of the proof of Part (a), one can easily see that $\Theta_{\lambda}:=\lim _{\varepsilon \downarrow 0} \theta_{[\lambda, \varepsilon]} \gg 0$ is well defined and that it provides us with a positive solution of the porous medium equation (i.e., (1.2) with $\Omega_{m}=\Omega$ ). This concludes the proof.

\section{References}

[1] A. G. Aronson \& L. A. Peletier, Large time behaviour of solutions of some porous medium equation in bounded domains, J. Diff. Eqns. 39 (1981), 378-412.

[2] G. I. Barenblatt, On some unsteady motions of a liquid or a gas in a porous medium, Prikl. Mat. Meh. 16 (1952), 67-68.

[3] H. Brezis \& L. Oswald, Remarks on sublinear elliptic equations, Nonl. Anal. T.M.A. 10 (1986), 55-64.

[4] M. Delgado, J. López-Gómez \& A. SuÁrez, Non-linear versus linear diffusion. From classical solutions to metasolutions, Adv. Diff. Eqns. 7 (2002), 1101-1124.

[5] P. Hess, On the solvability of nonlinear elliptic boundary value problems, Ind. Univ. Math. J., 25 (1976) 461-466.

[6] P. Hess \& T. Kato, On some linear and nonlinear eigenvalue problems with an indefinite weight function, Comm. Part. Diff. Eqns., 5 (1980), 999-1030.

[7] T. KAto, Perturbation Theory for Linear Operators, Classics in Mathematics, Springer, Berlin, 1995.

[8] J. LóPEz-Gómez, The maximum principle and the existence of principal eigenvalues for some linear weighted boundary value problems, J. Diff. Eqns., 127 (1996) 263294. 
[9] D. Sattinger, Topics in Stability and Bifurcation Theory, Lectures Notes in Mathematics, 309, Springer, Berlin, 1973. 